\title{
Review of Persistent Phrenic Nerve Palsy
}

\section{Andrew T Koogler* and Michael Kushelev}

Department of Anesthesiology, The Ohio State University, Wexner Medical Center, USA

\begin{abstract}
Regional anesthesia techniques for orthopedic surgeries have become more frequently utilized for postoperative analgesia. A commonly used technique for shoulder surgery is the interscalene brachial plexus nerve block which involves anesthetizing nerves of the shoulder. Unfortunately, patients can experience a transient phrenic nerve block resulting in dyspnea. Rarely this develops into a persistent phrenic nerve block that can out last the duration of the local anesthetics deposited around the brachial plexus. Patients with a persistent phrenic nerve block can present to neurologists and other specialists with symptoms that appear to have a cardiac or pulmonary etiology requiring prompt diagnosis and management.
\end{abstract}

Keywords: Persistent phrenic nerve palsy; PPNP; Interscalene brachial plexus block; Interscalene block; ISB

\section{Introduction}

Regional analgesia for arthroscopic or open surgical procedures requires accounting of the cutaneous, osseous and capsular innervation of the shoulder. A commonly used regional anesthetic technique, the interscalene block (ISB), anesthetizes the innervating nerves of the shoulder, with the goal of local anesthetic deposition in the vicinity of the C5-C6 nerve roots between the anterior and middle scalene muscle, also known as the interscalene groove. Besides optimizing postoperative pain, ISB can decrease patients' recovery time in the postanesthesia care unit and length of hospital stay [1]. Targeting the C5-C6 nerve roots allows analgesia of the axillary and suprascapular nerves which are primary in the innervation of shoulder joint. Additionally, the lateral pectoral, musculocutaneous and long thoracic nerves are anesthetized with the ISB providing complete anesthesia of shoulder [2].

The phrenic nerve originates from the third, fourth and fifth cervical ventral rami with some contribution from the cervical sympathetic ganglia or thoracic sympathetic plexus [3]. It originates near the roots of the brachial plexus, and courses over the lateral border of the anterior scalene muscle, posterior to the sternocleidomastoid muscle. The ISB is typically performed at the level of the cricoid cartilage (C5-C6), at which point the phrenic nerve is in near proximity (on average 1.8 $\mathrm{mm}$ ) medially to the brachial plexus. The phrenic nerve continues in an inferiomedial direction, and with each centimeter it courses caudally in the neck it creates an additional $3 \mathrm{~mm}$ of separation from the brachial plexus [4].

\section{Discussion}

Given the close proximity of the phrenic nerve and the brachial plexus at the interscalene groove, transient phrenic nerve palsy can be expected in up to $100 \%$ of patients with return to baseline following the resolution of the ISB, typically within $24 \mathrm{~h}$ [5]. Despite the near universal transient phrenic nerve palsy, persistent phrenic nerve palsies (PPNP) is a rare complication of ISB that has recently become more recognized in the medical literature. The range of PPNP reported varies from $0.048 \%$ to $1 \%$, with the true incidence difficult to determine likely to delays in diagnosis and under recognition [6,7].

Shoulder surgery can be complicated by nerve injury through direct trauma, excessive shoulder retraction, hematoma formation, or neck malpositioning during surgical manipulation. Most common injuries related to surgical factors involve the axillary or musculocutaneous nerves, occurring in $0.6 \%$ to $3.6 \%$ of shoulder arthroplasties [8]. More specifically, PNPP has been reported in a patient undergoing total shoulder arthroplasty without regional anesthesia. Patient positioning and retraction appear to play a crucial role in development of brachial plexus neuropathies, and possibly in the development of PPNP, as traction on the plexus is produced when the arm is abducted at or near 90 degrees, externally rotated and slightly extended for glenoid exposure during the long deltopectoral approach [9]. Medications, such as methotrexate, that modify metabolic processes and thus influence a nerve's conduction may also play a role in predisposing to nerve injuries in these patients [10]. Additionally, ISB can be complicated by nerve injuries such as PPNP by a variety of mechanisms including compression neuropathy due to direct needle trauma, intraneural injection, chemical toxicity, and neuronal ischemia [11-14]. Additional proposed mechanisms of PPNP include the "double crush" syndrome when two or more distinct compression sites occur along the course of a peripheral nerve. One of those insults can be related to the regional anesthetic while the second may either be a more proximal or distal compression related to surgery or underlying anatomy. These compression sites are thought to synergistically increase symptom intensity resulting a peripheral nerve neuropathy [15]. The proposed "triple crush" mechanism relates to the phrenic nerve compression by large volume injectate of local anesthetic around the brachial plexus in addition to the two or more distinct compression locations on the nerve [16]. Cervical degenerative disc is the only known patient risk factor for PPNP which may act as one of these distinct compression sites for both the "double crush" and "triple crush" mechanism of injury [6]. Definitive mechanism of action for PPNP is impossible to determine without a histological examination of the phrenic nerve.

Regional anesthesia, including ISB, is mostly performed with the assistance of "live" ultrasound (US) imaging of anatomic structures allowing visualization of nerve structures, blood vessels, and muscle tissue in relation to needle advancement. The goal of US guidance is to minimize trauma and optimally apply local anesthetics in relation to the targeted nerve structures. Prior to ubiquitous utilization of US, ISBs were performed with a combination of nerve stimulation and

*Corresponding author: Andrew T Koogler, MD, Department of Anesthesiology, The Ohio State University Wexner Medical Center, 410 W. 10th Ave., Columbus, OH 43210, USA, Tel: +1-614-271-1830; E-mail: andrew.koogler@osumc.edu

Received May 20, 2018; Accepted June 20, 2018; Published June 27, 2018

Citation: Koogler AT, Kushelev M (2018) Review of Persistent Phrenic Nerve Palsy Int J Neurorehabilitation 5: 319. doi: 10.4172/2376-0281.1000319

Copyright: (c) 2018 Koogler AT, et al. This is an open-access article distributed under the terms of the Creative Commons Attribution License, which permits unrestricted use, distribution, and reproduction in any medium, provided the original author and source are credited. 
paresthesia techniques. Although US guided techniques have not conclusively shown to decrease the risk of nerve injury related to regional anesthesia; US guided ISB provides anesthesiologists a better chance of identifying the phrenic nerve, decreases the number of needle passes and thus theoretically limiting trauma [17]. US targeted ISB focusing on decreasing total local anesthetic dose (lower volume and/ or concentration) administered or injecting further away from the C5C6 nerve roots have shown mixed results in decreasing the incidence of transient phrenic nerve palsy, but none have demonstrated a decrease, let alone elimination of the risk of PPNP [4,18-24].

Transient dyspnea is typically a primary complaint with short term phrenic nerve palsy due to absent caudal movement of the diaphragm resulting in impaired ventilation and lower lung volumes [2]. Additionally, long standing PPNP can reduce perfusion to the ipsilateral basal portion of the lung from possible hypoxia induced vasoconstriction, creating a ventilation/perfusion (V/Q) mismatch. Poor chest wall and pulmonary compliance in combination with the V/Q mismatch results in symptomatic dyspnea and possible hypoxemia. Patients may also experience orthopnea from abdominal content pressure upon the ipsilateral lung. Left PPNP and resultant hemidiaphragmatic paralysis can produce gastrointestinal-like symptoms such as heartburn, regurgitation, nausea, epigastric pain, bloating and constipation [25]. Accessory and intercostal muscle contraction to help expand the rib cage can allow healthy patients to maintain normal lung volumes. Patients with pre-existing lung disease or obesity are more likely to experience long term dyspnea, hypoxia and may require supplemental oxygen or even ventilatory support [2]. Reductions in lung volume in patients with PPNP can lead to the development of obstructive lung disorders, respiratory infections, and sleep-disordered breathing requiring positive pressure ventilation $[26$, 27].

Phrenic nerve palsy can be diagnosed with multiple different studies, such as chest $\mathrm{x}$-ray, diaphragm fluoroscopy (sniff test), spirometry (pulmonary function testing), nerve conduction testing and electromyography (EMG) $[2,28]$. Hemidiaphragmatic elevation on the ipsilateral side of the affected phrenic nerve would be expected in chest $\mathrm{x}$-ray imaging. Diaphragm elevation, absence of inspiratory motion, or paradoxical movement would be expected and necessary to diagnose phrenic nerve palsy on diaphragmatic fluoroscopy. Comparing isolated lung function to predicted values with spirometry can be utilized for diagnosis, but if available comparisons to baseline function is more accurate. Total lung capacity (TLC), forced vital capacity (FVC), inspiratory capacity, and forced expiratory volume in 1s (FEV1), and peak expiratory flow rates are all reduced in phrenic nerve palsy after ISB [2]. Nerve conduction studies and EMG can be performed to assess volitional respiratory efforts. Diaphragmatic voluntary motor units would be expected to be absent in phrenic nerve palsy [28].

PPNP resolution can take up to 24 months after the initial injury with conservative treatment such as physical therapy and close follow up. The long course and slow rate of nerve regeneration owes to the extended recovery period for the phrenic nerve [29]. Surgical treatments are an option for patients with PPNP who have not subjectively or objectively improved with conservative management. Diaphragm plication is a long standing surgical option that can be performed open or minimally invasive via transthoracic or transabdominal approaches. The goal of diaphragmatic plication is to improve symptoms of dyspnea by surgically lowering the elevated hemidiaphragm and securing it in a more caudal position to allow expansion of the ipsilateral lung. One month postoperatively patients have improvement in FEV1, FVC and maximum forced inspiratory flow; however, patients do not seem to progressively improve beyond that time period [25]. The surgical approach for phrenic nerve reconstruction surgery varies based on location of the site of the phrenic injury. Phrenic nerve reconstruction surgery consists of locating the site of injury and subsequent separation of the cervical roots and phrenic nerve from musculofascial and vascular adhesions via microsurgical neurolysis decompression. In order to preserve function of a nearby donor nerve, redundant nerve branch or end-to-side grafting methods are used during nerve interposition grafting and/or neurotization. To prevent recurrence of adhesions postoperatively, collagen nerve wraps are positioned around both the phrenic nerve and nerve grafts. Thoracic phrenic nerve injury reconstruction is a combined surgical effort of thoracic and reconstructive nerve surgeons. The approach consists of videoassisted thoracoscopic (VATS) or minithoracotomy performed by a thoracic surgeon followed by nerve decompression and reconstruction by the nerve reconstruction surgeons. Following nerve reconstruction patients need to enhance muscle recovery after surgery with diaphragm retraining therapy. These patients can have a $37 \%$ improvement at 1 year and up to $125 \%$ improvement after 2 years in their diaphragm motor amplitudes after surgical reconstruction. Patients with a "complex" phrenic nerve injury can also have diaphragmatic pacemaker insertion. These patients would include remote injuries ( $>10$ years with intact motor units), bilateral diaphragmatic dysfunction, or those having previous radiation and/or extensive scaring in the cervical and thoracic regions [28].

\section{Conclusion}

Patients with dyspnea, elevated hemidiaphragm and undiagnosed PPNP may be referred to neurologists for further evaluation and management following shoulder surgery. Unfortunately, many of the signs and symptoms in PPNP patients are nonspecific and their dyspnea may appear to have a cardiac or pulmonary etiology. While the mechanism for PPNP may be difficult to isolate, the potential relation to ISB should be considered. A thorough history of surgical and regional anesthesia procedures, especially ISB, should be sought in order to guide possible diagnostic testing and treatment in these patients.

\section{References}

1. Bryan NA, Swenson JD, Greis PE, Burks RT (2007) Indwelling interscalene catheter use in an outpatient setting for shoulder surgery: Technique, efficacy and complications. J Shoulder Elbow Surg 16: 388-395.

2. El-Boghdadly K, Chin KJ, Chan VWS (2017) Phrenic nerve palsy and regional anesthesia for shoulder surgery: Anatomical, physiologic and clinical considerations. Anesthesiology 127: 173-191.

3. Verin E, Marie JP, Similowski T (2011) Cartography of human diaphragmatic innervation: Preliminary data. Respir Physiol Neurobiol 176: 68-71.

4. Kessler J, Schafhalter-Zoppoth I, Gray AT (2008) An ultrasound study of the phrenic nerve in the posterior cervical triangle: Implications for the interscalene brachial plexus block. Reg Anesth Pain Med 33: 545-550.

5. Urmey WF, Talts KH, Sharrock NE (1991) One hundred percent incidence of hemidiaphragmatic paresis associated with interscalene brachial plexus anesthesia as diagnosed by ultrasonography. Anesth Analg 72: 498-503.

6. Pakala SR, Beckman JD, Lyman S, Zayas VM (2013) Cervical spine disease is a risk factor for persistent phrenic nerve paresis following interscalene nerve block. Reg Anesth Pain Med 38: 239-242.

7. Jules-Elysee K, Reid SC, Kahn RL, Edmonds CR, Urban MK (2014) Prolonged diaphragm dysfunction after interscalene brachial plexus block and shoulder surgery: A prospective observational pilot study. Br J Anaesth 112: 950-951.

8. Dwyer T, Henry PDG, Cholvisudhi P, Chan VW, Theodoropoulos JS, et al. (2015) Neurological complications related to elective orthopedic surgery: Part 1: Common shoulder and elbow procedures. Reg Anesth Pain Med 40: 431-442. 
9. Lynch NM, Cofield RH, Silbert PL, Hermann RC (1996) Neurologic complications after total shoulder arthroplasty. J Shoulder Elbow Surg 5: 53-61.

10. Sunderland S (1951) A classification of peripheral nerve injuries producing loss of function. Brain 74: 491-516.

11. Deruddre S, Vidal D, Benhamou D (2006)A case of persistent hemidiaphragmatic paralysis following interscalene brachial plexus block. J Clin Anesth 18: 238239.

12. Ediale KR, Myung CR, Neuman GG (2004) Prolonged hemidiaphragmatic paralysis following interscalene brachial plexus block. J Clin Anesth 16: 573575

13. Robaux S, Bouaziz H, Boisseau N, Raucoules-Aimé M, Laxenaire MC (2001) Persistent phrenic nerve paralysis following interscalene brachial plexus block. Anesthesiology 95: 1519-1521.

14. Bashein G, Robertson HT, Kennedy WF Jr (1985) Persistent phrenic nerve paresis following interscalene brachial plexus block. Anesthesiology 63: 102104.

15. Kane P, Daniels A, Akelman E (2015) Double crush syndrome. J Am Acad Orthop Surg 23: 558-562.

16. De A, Hayes JE (2013) Persistent phrenic nerve paresis after interscalene block: A "triple crush" hypothesis of nerve injury. Reg Anesth Pain Med 38: 553.

17. Neal JM, Barrington MJ, Brull R, Hadzic A, Hebl JR, et al. (2015) The second ASRA Practice Advisory on neurologic complications in regional anesthesia and pain medicine. Reg Anesth Pain Med 40: 401-430.

18. Prates AG Jr, Vasques LC, Bordoni LS (2015) Anatomical variations of the phrenic nerve: An actualized review. J Morphol Sci 32: 53-56.

19. Koogler AT, Kushelev M (2018) Ultrasound-guided interscalene catheter complicated by persistent phrenic nerve palsy. Case Rep Anesthesiol 2018 9873621.

20. Riazi S, Carmichael N, Awad I, Holtby RM, McCartney CJ (2008) Effect of local anaesthetic volume (20 vs $5 \mathrm{ml}$ ) on the efficacy and respiratory consequences of ultrasound-guided interscalene brachial plexus block. Br J Anaesth 101: 549556.

21. Lee JH, Cho SH, Kim SH, Chae WS, Jin HC, et al. (2011) Ropivacaine for ultrasound-guided interscalene block: $5 \mathrm{~mL}$ provides similar analgesia but less phrenic nerve paralysis than $10 \mathrm{~mL}$. Can J Anaesth 58: 1001-1006.

22. Wong AK, Keeney LG, Chen L, Williams R, Liu J, et al. (2016) Effect of local anesthetic concentration $(0.2 \%$ vs $0.1 \%$ ropivacaine $)$ on pulmonary function and analgesia after ultrasound-guided interscalene brachial plexus block: A randomized controlled study. Pain Med 17: 2397-2403.

23. Thackeray EM, Swenson JD, Gertsch MC, Phillips KM, Steele JW, et al. (2013) Diaphragm function after interscalene brachial plexus block: A double-blind, randomized comparison of $0.25 \%$ and $0.125 \%$ bupivacaine. J Shoulder Elbow Surg 22: 381-386.

24. Zhai W, Wang X, Rong Y, Li M, Wang H (2016) Effects of a fixed low-dose ropivacaine with different volume and concentrations on interscalene brachial plexus block: A randomized controlled trial. BMC Anesthesiol 16: 80.

25. Groth SS, Andrade RS (2010) Diaphragm plication for eventration or paralysis A review of the literature. Ann Thorac Surg 89: S2146-S2150.

26. Patterson DL, DeRemee RA, Hunt LW (1994) Severe asthma complicated by bilateral diaphragmatic paralysis attributed to Parsonage-Turner syndrome. Mayo Clin Proc 69: 774-778.

27. Khan A, Morgenthaler TI, Ramar K (2014) Sleep disordered breathing in isolated unilateral and bilateral diaphragmatic dysfunction. J Clin Sleep Med 10: $509-515$

28. Kaufman MR, Elkwood Al, Brown D, Cece J, Martins C, et al. (2017) Longterm follow-up after phrenic nerve reconstruction for diaphragmatic paralysis: $A$ review of 180 patients. J Reconstr Microsurg 33: 63-69.

29. Wilcox PG, Paré PD, Pardy RL (1990) Recovery after unilateral phrenic nerve injury associated with coronary artery revascularization. Chest 98: 661-666. 